\title{
Implementation of the Local Reference Node Concept for Spatially Distributed Circuits
}

\author{
Carlos E. Christoffersen, ${ }^{1}$ Michael B. Steer ${ }^{2}$
}

${ }^{1}$ Department of Electrical and Computer Engineering, North Carolina State University, Raleigh, North Carolina 27695-7914, U.S.A.

${ }^{2}$ Institute of Microwaves and Photonics, Department of Electronic and Electrical Engineering, University of Leeds, Leeds LS2 9JT, United Kingdom.

ABSTRACT: Large spatially distributed microwave and millimeter-wave systems involve the interaction of linear and nonlinear circuits with their electromagnetic environment. Here a circuit-level approach to the modeling of circuit-field interactions is developed.

Keywords: modified nodal analysis; circuit simulation; quasi-optical systems; spatially distributed circuits; local reference node

\section{INTRODUCTION}

The distributed nature of many microwave and millimeter-wave circuits necessitates electromagnetic modeling. Thus the full application of computer-aided engineering (CAE) to these circuits requires the integration of electromagnetic models of distributed structures with conventional circuit analysis. In the formative stages of the CAE of microwave circuits, ports were used in specifying the interconnectivity of networks. The utilization of ports avoided the issue of specifying reference nodes. In analysis, using matrix manipulations or signal flow graphs, one of the terminals of a port was used implicitly as a reference node and generally ignored in formulating the mathematical model. In one commercial nonlinear microwave simulator ports are still used in circuit specification. The connection of discrete elements is specified nodally but at the highest level of the hierarchy port-based descriptions are used. While it is possible to analyze any circuit with this arrangement it becomes increasingly difficult to specify the connections of large spatially distributed circuits, and also to specify and extract desired output quantities. The alternative to using a port-only descriptions is to exclusively use the nodal connectivity description - the only method used in general purpose circuit simulators. The conventional nodal specification enables circuit elements to be connected in any possible combination and only one reference node (commonly called the global reference node or simply ground) is used. So nodal specification provides tremendous flexibility in circuit specification but the multiple reference nodes leads to $\mathrm{M}$ fold indefiniteness of the (modified) nodal admittance matrix. Also, with spatially distributed circuits it is possible to make illegal connections such as connecting a non-spatially distributed element, say a resistor, across a spatially distributed element, e.g. a transmission line. The purpose of this paper is two-fold: firstly to describe an implementation of the local reference node theory described by Khalil and Steer in Reference [7]. Secondly to develop and describe the implementation of a scheme for checking the legal connections of a network with mixed spatially distributed and conventional elements. 


\section{BACKGROUND}

A circuit is a graphical construct (which can be specified in textual form as a netlist) for coupling together algebraic and first order differential equations. These equations arise from the constitutive relations of the individual elements, which specify the actual form of the individual equations, and from Kirchoff's current law which specifies how the equations are coupled. With but a few modifications, the analysis thus described is called nodal admittance analysis. It is important to note that circuits have no sense of space - a circuit is defined as though it existed at a point. The problem is then how to incorporate an electromagnetic model of a structure that is inherently distributed in space. Of two approaches, one is to insert the device equations into an appropriate time-stepping electromagnetic simulator such as a finite difference time domain (FDTD) simulator $[1,2]$. This reduces the level of abstraction of the "circuit" and embeds the constitutive relations of the conventional circuit elements into the analysis grid of the FDTD method. An alternative is to retain the high-level circuit abstraction and incorporate the results of a field analysis (the spatially distributed circuit) into a circuit structure $[3,4,5,6]$. Thus the problem is one of taking an electromagnetic solution and using a physically consistent approach to inserting it is a circuit element. This is a less direct approach, that is more abstract, but potentially much more general and compatible with the large body of existing computer-aided circuit analysis theory and knowledge that has been developed.

At this point it is useful to review modified nodal admittance (MNA) analysis to indicate the basis for the work presented here. MNA analysis was developed to handle elements that do not have nodal admittance descriptions. For each such element one or more additional equations are added to the nodal admittance equations and these new equations become additional rows and columns in the evolving matrix system of equations. A similar approach can be followed for the electromagnetic elements. The process is a little more sophisticated as it is no longer sufficient to add additional rows. Instead the concept of local reference nodes was developed [7] as a generalization of the compression matrix approach [6]. This concept provides another way to incorporate alternate equations in the evolving MNA matrix. However, rather than adding additional constitutive relations, the local reference node concept changes the way the port-based parameters are used. Figure 2 shows a circuit with a spatially distributed circuit and with local reference nodes indicated by the diagonal symbol. In a conventional circuit only one reference node (ground) is possible so that application of KCL to the global reference node introduces just one additional redundant row and column in the indefinite form of the MNA matrix. For a spatially distributed circuit, KCL is applied to each locally referenced group one at a time and, as the local reference nodes are electrically connected only through a spatially distributed element, each application results in a redundant row and column in the MNA matrix. This is a particular property of the port-based characterization of the spatially distributed element [7]. Thus only appropriate electromagnetic analyses are suitable. The mathematical description of the required extension to the nodal admittance analysis is given in [7]. 


\section{METHOD}

The basis of the method is that in a multi-port element, the terminals of ports with different local reference nodes can be considered isolated. For example, if we write the equivalent circuit of a two-element derived from the port-based admittance $(y)$ parameters,

$$
\left[\begin{array}{l}
i_{1} \\
i_{2}
\end{array}\right]=\left[\begin{array}{ll}
y_{11} & y_{12} \\
y_{21} & y_{22}
\end{array}\right]\left[\begin{array}{l}
v_{1} \\
v_{2}
\end{array}\right]
$$

we obtain the equivalent circuit in Figure 1. Note that no current can flow between the two ports. This type of circuit model can be generalized for an n-port element where external and internal local reference nodes are defined. The local references shown in Figure 1 are internal because they are used internally in the element to measure the voltages at its ports. An external local reference terminal, on the other hand, is an arbitrarily chosen terminal from a locally referenced group. In general, for any spatially distributed circuit (Figure 2) there is no current between port groups inside the spatially-distributed element. Thus the circuit can be divided into subcircuits, each with a local reference terminal, as shown in Figure 3. Each subcircuit is isolated with respect to the others, so there is no change in the solution if all the reference nodes are connected together. The difference is that now there is only one global reference node for all the circuit, and standard nodal methods can be used to formulate the circuit equations. The problem becomes that of detecting violations to the assumption that each locally referenced group is isolated from the others and that there is exactly one reference terminal for each one. Such a situation is shown in Figure 4. The effect of this connection is the creation of a current loop that is non-physical since the two circuits cannot be connected instantaneously. On the other hand, it is valid for two subcircuits to be connected by more than one spatially distributed element. It is also possible for two ports of a spatially distributed element to be connected to the same locally referenced group (for example a delay line). In that case, the external local reference node would be the same for both ports of the line, but internally, the line still has two local reference nodes.

After all the checking is done, all the local reference nodes are merged into a single global reference node, and the solution of the circuit is found by standard procedures. The nodal voltages found are coincident with the voltages referred to each local reference node.

\section{IMPLEMENTATION}

Implementation of the technique can be described using a conventional procedural approach or an object oriented approach. While not obvious to those not conversant with object oriented practice, the object oriented view maps onto the circuit analysis problem cleanly. A circuit is a collection of objects (resistors, inductors, transmission lines, etc.) that are related to each other (they share nodes). With the introduction of spatially distributed elements along with the conventional elements there are certain rules that describe allowable interconnections. The rule is

Spatially distributed objects can be connected together in any manner but the interconnections of non-spatially distributed elements cannot span spatially distributed elements. 
In Transim (our simulator), each element and each terminal is a node in a graph structure. The algorithm to detect violations is a variation of the depth-search algorithm and is best illustrated using what is called a collaboration diagram. The algorithm could also be implemented using conventional procedural programming [8]. The collaboration diagram [9] is part of the Unified Modeling Language (UML), a language for specifying, visualizing, and constructing the artifacts of software systems as well as for business modeling. The UML represents a collection of "best engineering practices" that have proven successful in the modeling of large and complex systems. Behavior is implemented by sets of objects that exchange messages within an overall interaction to accomplish a purpose. To understand the mechanisms used in a design, it is important to see only the objects and the messages involved in accomplishing a purpose or a related set of purposes, projected from the larger system of which they are part for other purposes. Such a static construct is called a collaboration. A collaboration is a set of participants and relationships that are meaningful for a given set of purposes. The identification of participants and their relationships does not have global meaning. In the collaboration diagram, each box represents an object (in this case, either a terminal or an element). The lines between boxes represent associations and the arrows are messages. The order in which messages are sent is shown by the numbers.

Figure 5 shows the circuit view corresponding to the collaboration diagram of Figure 6 . The terminal numbers indicate the order in which terminals are discovered by the algorithm. Note also that the internal local reference terminals of the spatially distributed element do not need to be coincident with the external references. The search begins at each local reference terminal. The local reference terminal sends a message to propagate its identifier (id) to all the adjacent elements. If the element that receive the message has only one local reference terminal (lumped), it continues the propagation to the other adjacent terminals. Otherwise it only propagates the id to the element terminals with the same internal reference. If an element attempts to propagate a reference id to a terminal already marked with another reference id, then a violation is detected: there is an illegal connection between two locally referenced groups. A check to detect floating parts of the circuit can be made by finding any terminal that does not belong to any locally referenced group. All these checks are implemented in Transim. Note that the bias circuit often violates the condition that the locally referenced groups are isolated between them. To overcome this either the distributed elements should include the DC characteristics or an independent DC source should be considered in each group. In the input netlist, the local reference nodes are identified with the command ".ref <terminal>". By default the nodes named "0" and "gnd" are assumed to be reference nodes.

\section{DISCUSSION}

The concepts and algorithms presented in this paper are well illustrated using a spatially distributed spatial power combining circuit $[10,11]$ as an example. The system is shown in Figure 7 where a signal transmitted by a horn antenna illuminates a $2 \times 2$ grid of amplifier unit cells. The planar array of amplifiers is shown in greater detail in Figure 8 where each unit cell is localized at a grid intersection and comprises two MMIC's in a push-pull configuration. Each unit cell is separated from its neighbors and each radiates amplified power at the orthogonal polarization to the input signal. The contributions from each cell combine spatially and the combined power is collected by the output horn antenna. The electromagnetic environment is modeled using Method of Moments (MOM) techniques [10]. Clearly a global reference node cannot be defined for this electrically large structure. Instead local reference nodes are defined, one per unit cell and one for each of the 
bias circuit connections, so that there are sixteen local reference nodes. The Spice-like netlist for this system is given in Figure 9. In this netlist there are three special element types, namely the ymatrix, gridex and vct. The ymatrix element is used to define a multi-port element described by its $y$ parameters. The information to build the element is stored in a file that contains the $y$ parameters as well as the port information. In this example, the element was used to model the quasi-optical grid and the element file was generated using an electromagnetic simulator called yomoma. The gridex is an element to provide the field excitation to the grid. From the circuit point of view it looks like a set of current sources. The input files were also generated by the yomoma MOM program [10]. The vct element is a nonlinear voltage-controlled current source. It is used inside the MMIC behavioral model. Note that the $y$ matrix defined element generated by yomoma set to zero the DC matrix. Therefore the bias circuit can not be modeled inside the spatially distributed element and must be implemented using standard lumped elements. This could lead to a violation of the condition that the locally referenced groups are isolated between them. To overcome this, either the distributed elements should include the DC characteristics or an independent bias circuit must be used in each locally referenced group.

\section{CONCLUSION}

The approach described here enables conventional circuit simulators to be extended to incorporate spatially distributed circuits. The implementation is an extension of modified nodal admittance analysis and the overhead involved in implementing the scheme is small. The authors have used the work described here for the steady-state modeling of quasi-optical power combining circuits [10, 11]. In conventional nodal-based analysis the row and column associated with the global reference node are not inserted into the evolving modified nodal admittance matrix. The same inspection procedure is used with locally referenced nodes. Thus the additional row and column associated with each locally referenced node are not incorporated into the evolving matrix equation and so avoiding the substantial performance penalty that would be incurred with explicit row and column elimination. If required these rows and columns can always be reconstructed by knowing the members of a locally referenced group [7]. The effective elimination of the one row and column per locally referenced leads to a non-singular matrix. 


\section{ACKNOWLEDGMENT}

This work was supported by the Defense Advanced Research Projects Agency (DARPA) through the MAFET Thrust III program as DARPA agreement number DAAL01-96-K-3619.

\section{REFERENCES}

1. S. M. S. Imtiaz and S. M. El-Ghazaly, "Global modeling of millimeter-wave circuits: electromagnetic simulation of amplifiers," IEEE Trans. on Microwave Theory and Tech., vol 45, pp. 2208-2217, Dec. 1997.

2. C.-N. Kuo, R.-B. Wu, B. Houshmand, and T. Itoh, Modeling of microwave active devices using the FDTD analysis based on the voltage-source approach, IEEE Microwave Guided Wave Lett., vol. 6, pp. 199-201, May 1996.

3. E. Larique, S. Mons, D. Baillargeat, S. Verdeyme, M. Aubourg, P. Guillon, and R. Quere, "Electromagnetic analysis for microwave FET modeling," IEEE microwave and guided wave letters Vol 8, pp. 41--43, Jan. 1998.

4. T. W. Nuteson, H. Hwang, M. B. Steer, K. Naishadham, J.W.Mink, and J. Harvey, "Analysis of finite grid structures with lenses in quasi-optical systems," IEEE Trans. Microwave Theory Techniques, pp. 666-672, May 1997.

5. M. B. Steer, M. N. Abdullah, C. Christoffersen, M. Summers, S. Nakazawa, A. Khalil, and J. Harvey, "Integrated electro-magnetic and circuit modeling of large microwave and millimeter-wave structures," Proc. 1998 IEEE Antennas and Propagation Symp., pp. 478481, June 1998.

6. J. Kunisch and I. Wolff, "Steady-state analysis of nonlinear forced and autonomous microwave circuits using the compression approach," Int. J. of Microwave and MillimeterWave Computer-Aided Engineering, vol. 5, No. 4, pp. 241-225, 1995

7. A. I. Khalil and M. B. Steer, "Circuit theory for spatially distributed microwave circuits," IEEE Trans. on Microwave Theory and Techniques, vol. 46, pp. 1500-1503, Oct. 1998.

8. T. H. Cormen, C. E. Leiserson and R. L. Rivest, Introduction to Algorithms, The MIT Press, McGraw-Hill Book Company, 1990.

9. Rational Software, UML Resources, http://www.rational.com/uml/documentation.html.

10. M. A. Summers, C. E. Christoffersen, A. I. Khalil, S. Nakazawa, T. W. Nuteson, M. B. Steer, and J. W. Mink, "An integrated electromagnetic and nonlinear circuit simulation environment for spatial power combining systems," Proc. 1998 IEEE MTT-S International Microwave Symp., June 1998, 1473-1476.

11. M. B. Steer, M. Ozkar and C. E. Christoffersen, "Circuit level modelling of spatially distributed mm and sub mm-Wave Systems," Proc. 1998 IEEE Sixth International Conference on Terahertz Electronics, September 1998, pp. 21-24. 


\section{CAPTIONS}

Figure 1. Equivalent circuit of a two-port distributed element.

Figure 2 Generic spatially distributed circuit.

Figure 3. The locally referenced groups are isolated, therefore the solution is unchanged if the local references are connected.

Figure 4. Illegal connection between groups.

Figure 5. Circuit containing an illegal element connection. The numbers at the nodes indicate the order in which terminals are discovered.

Figure 6. Collaboration diagram showing the violation detection algorithm.

Figure 7. A simple $2 \times 2$ spatial grid amplifier.

Figure 8. Local groups in a quasi-optical grid amplifier.

Figure 9. Transim netlist for the grid amplifier circuit of Figure 7. 


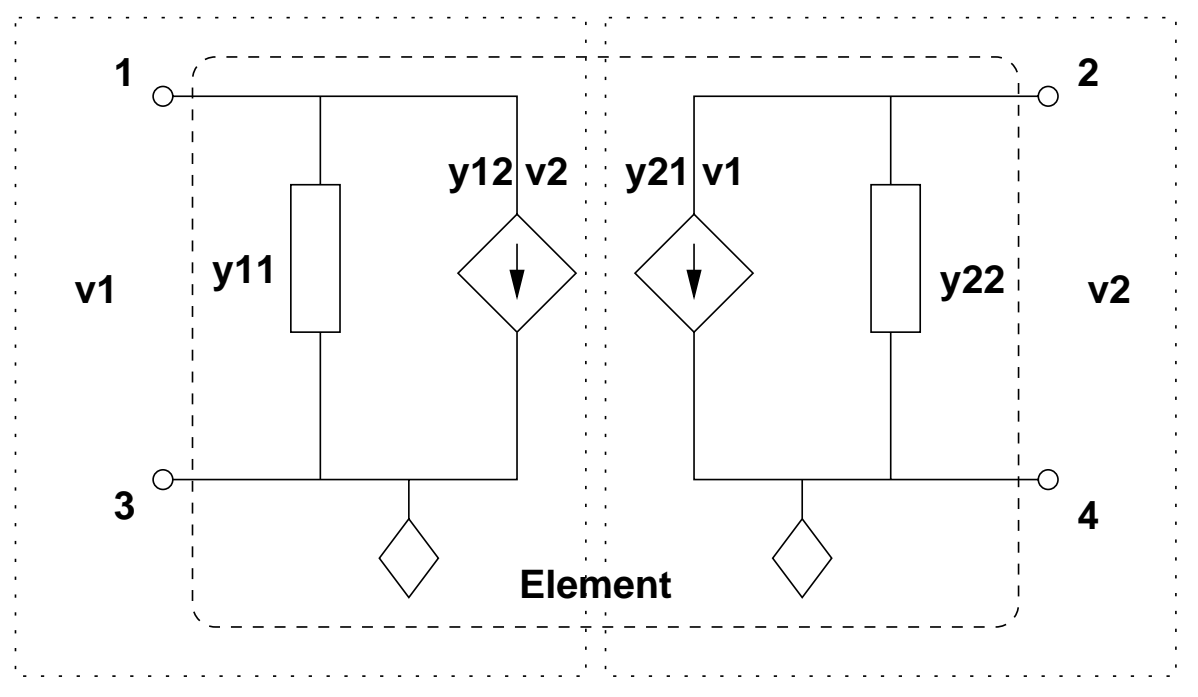

Figure 1. Equivalent circuit of a two-port distributed element. 

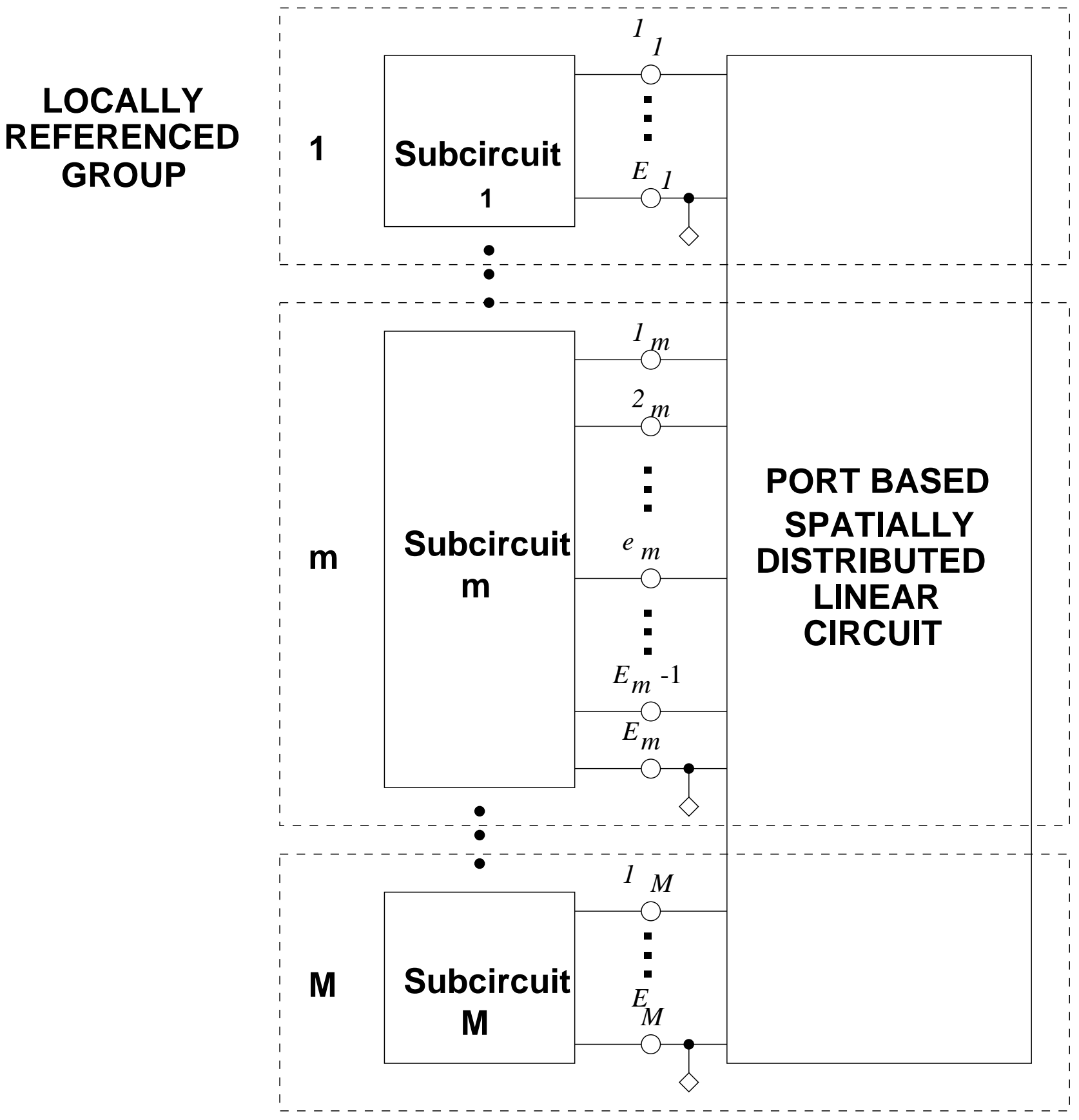

Figure 2 Generic spatially distributed circuit. 


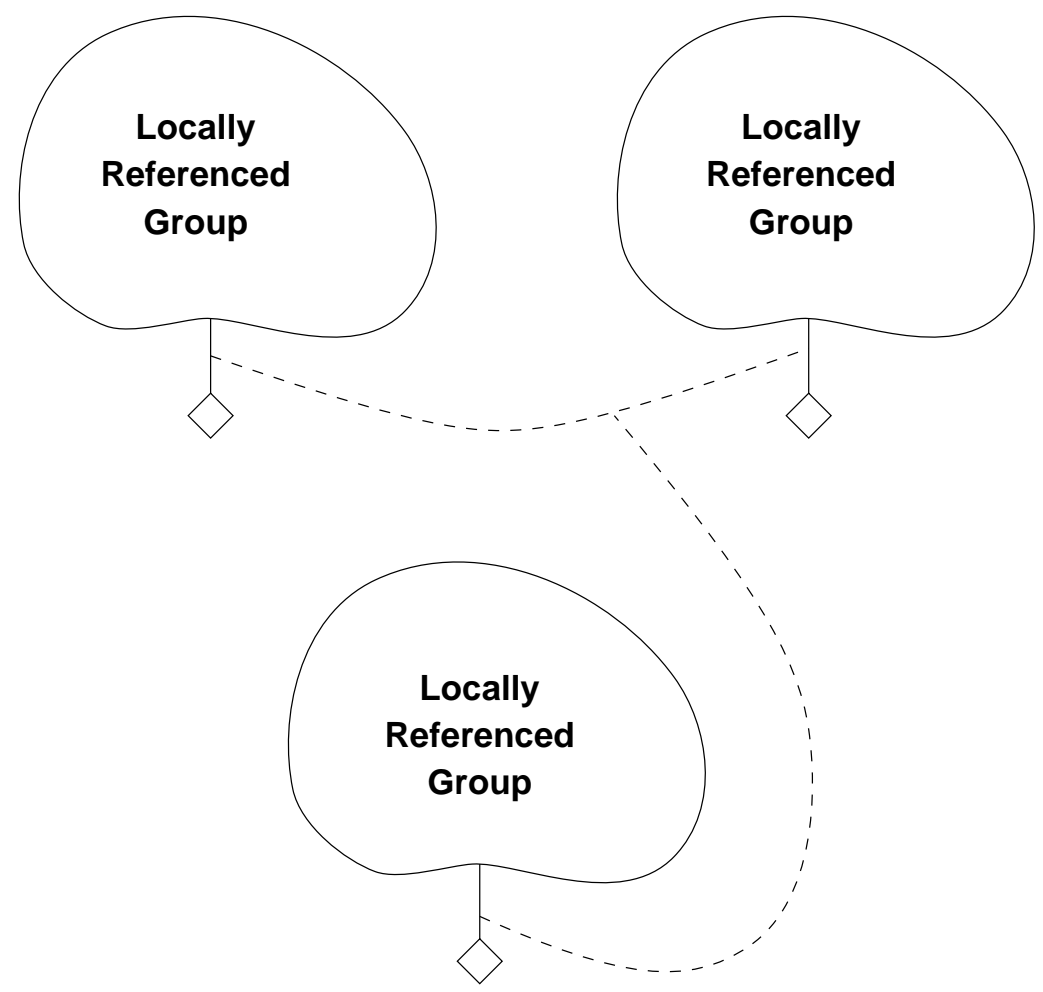

Figure 3. The locally referenced groups are isolated, therefore the solution is unchanged if the local references are connected.

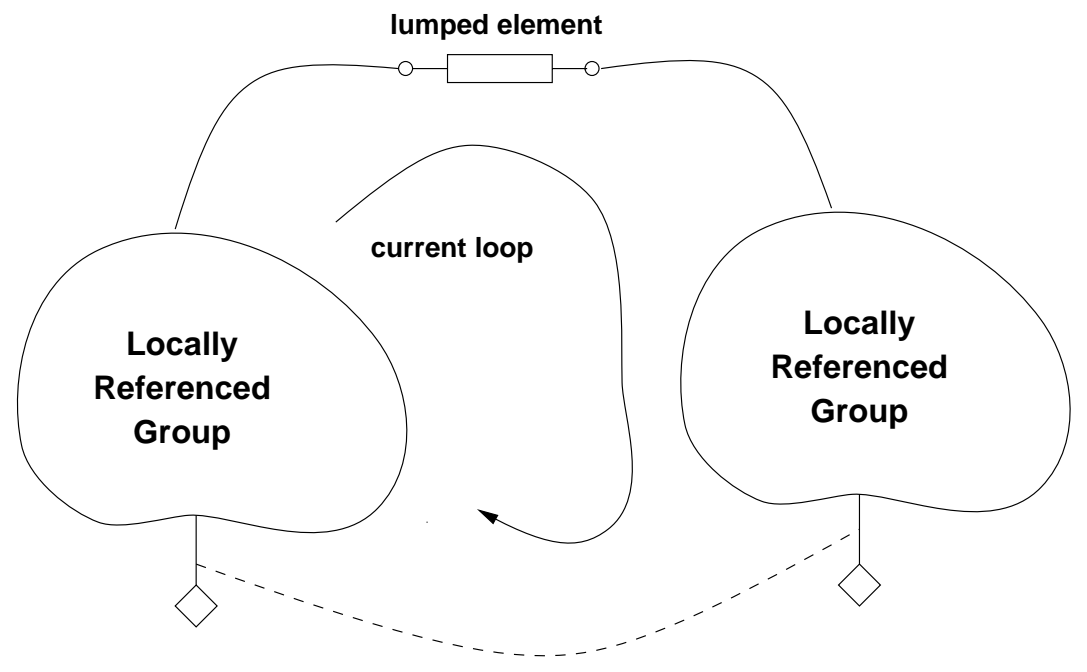

Figure 4. Illegal connection between groups. 


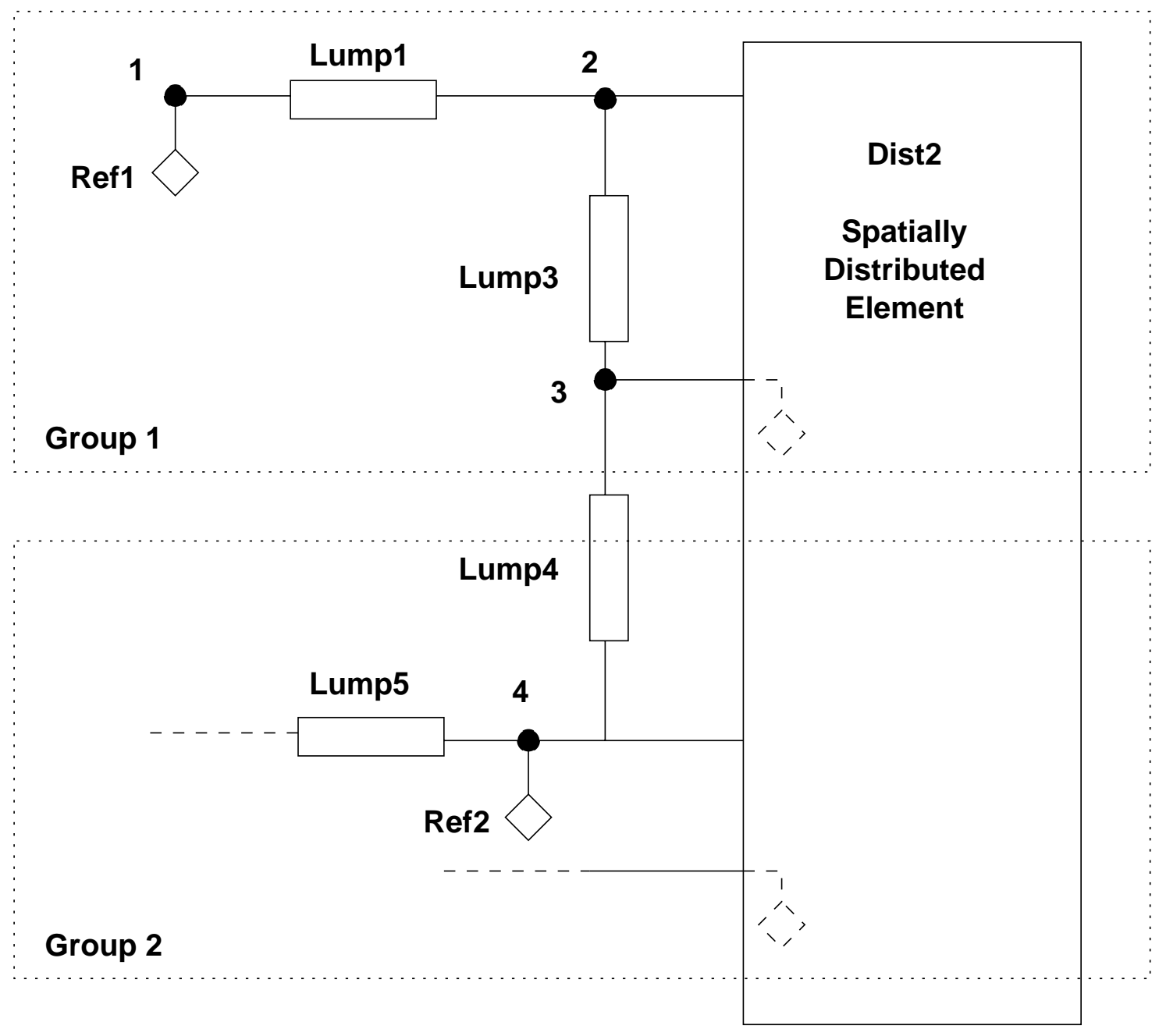

Figure 5. Circuit containing an illegal element connection. The numbers at the nodes indicate the order in which terminals are discovered. 


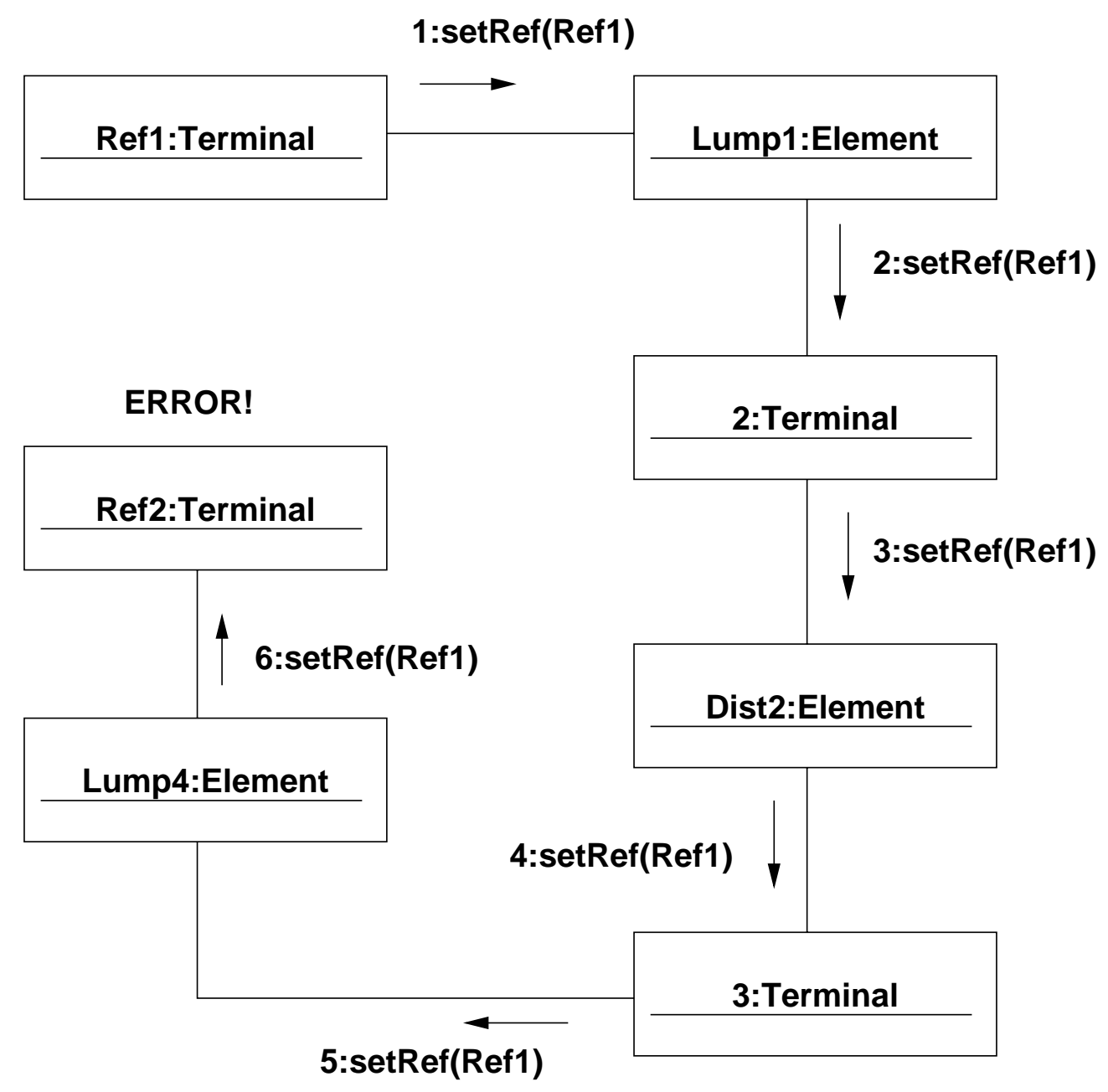

Figure 6. Collaboration diagram showing the violation detection algorithm. 

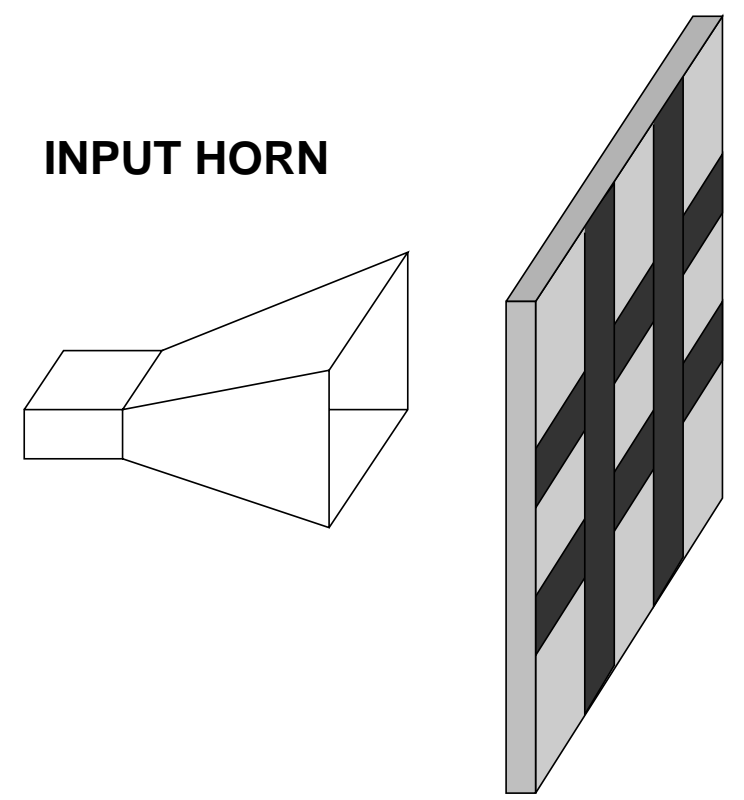

\section{OUTPUT HORN}

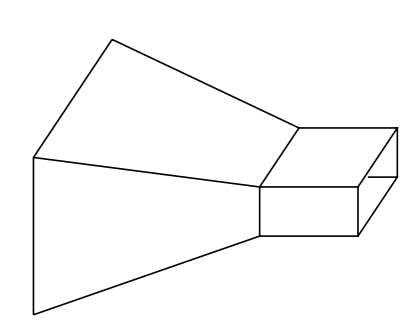

Figure 7. A simple 2x2 spatial grid amplifier.

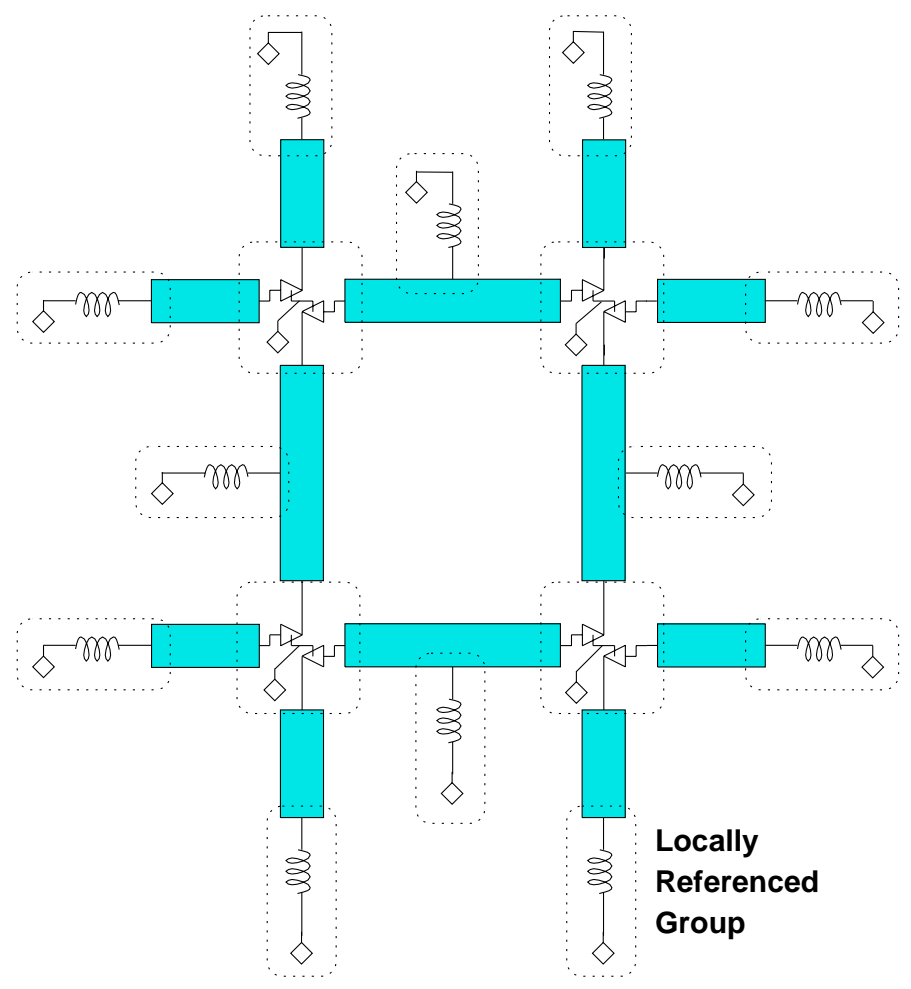

Figure 8. Local groups in a quasi-optical grid amplifier. 


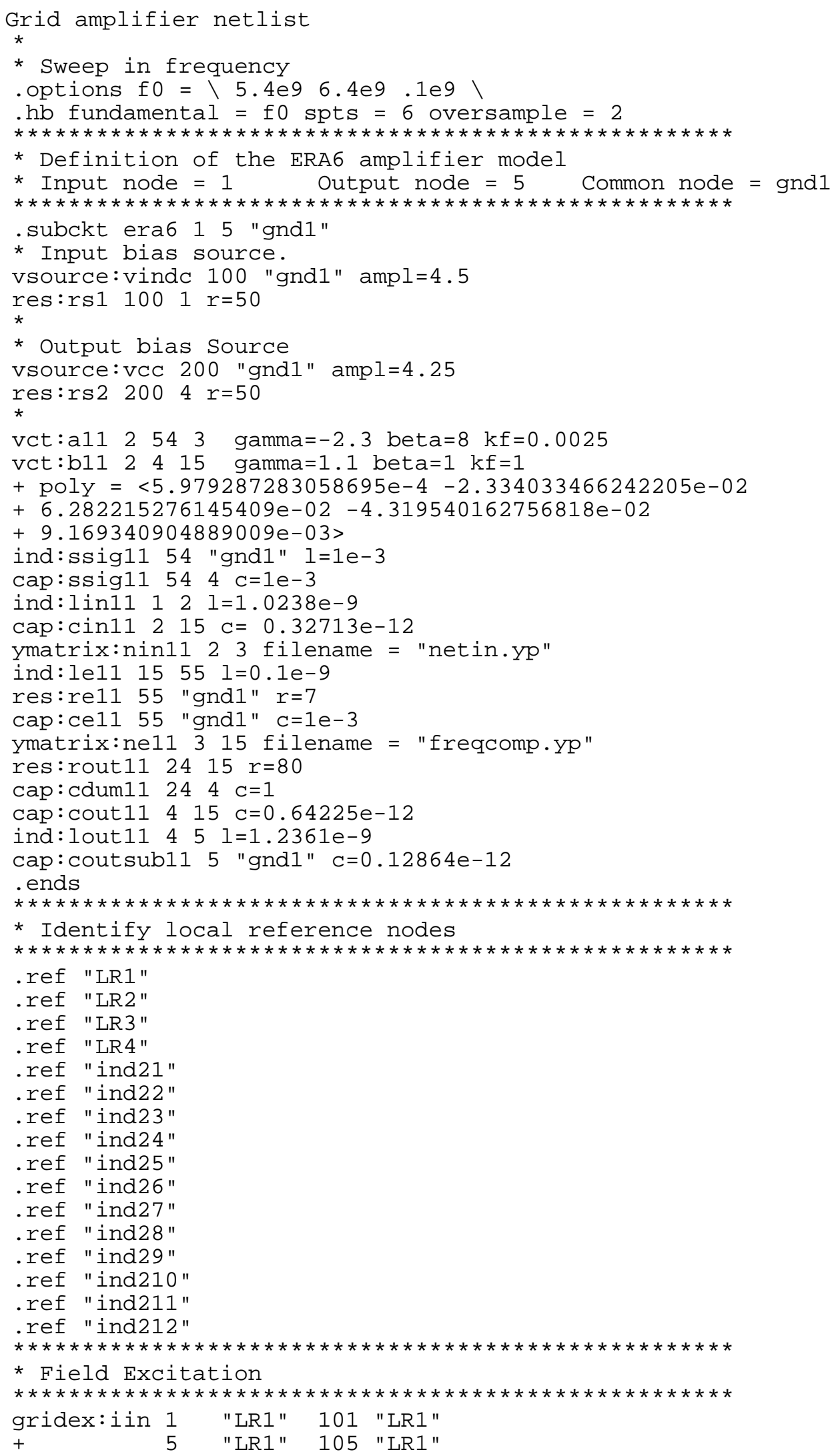




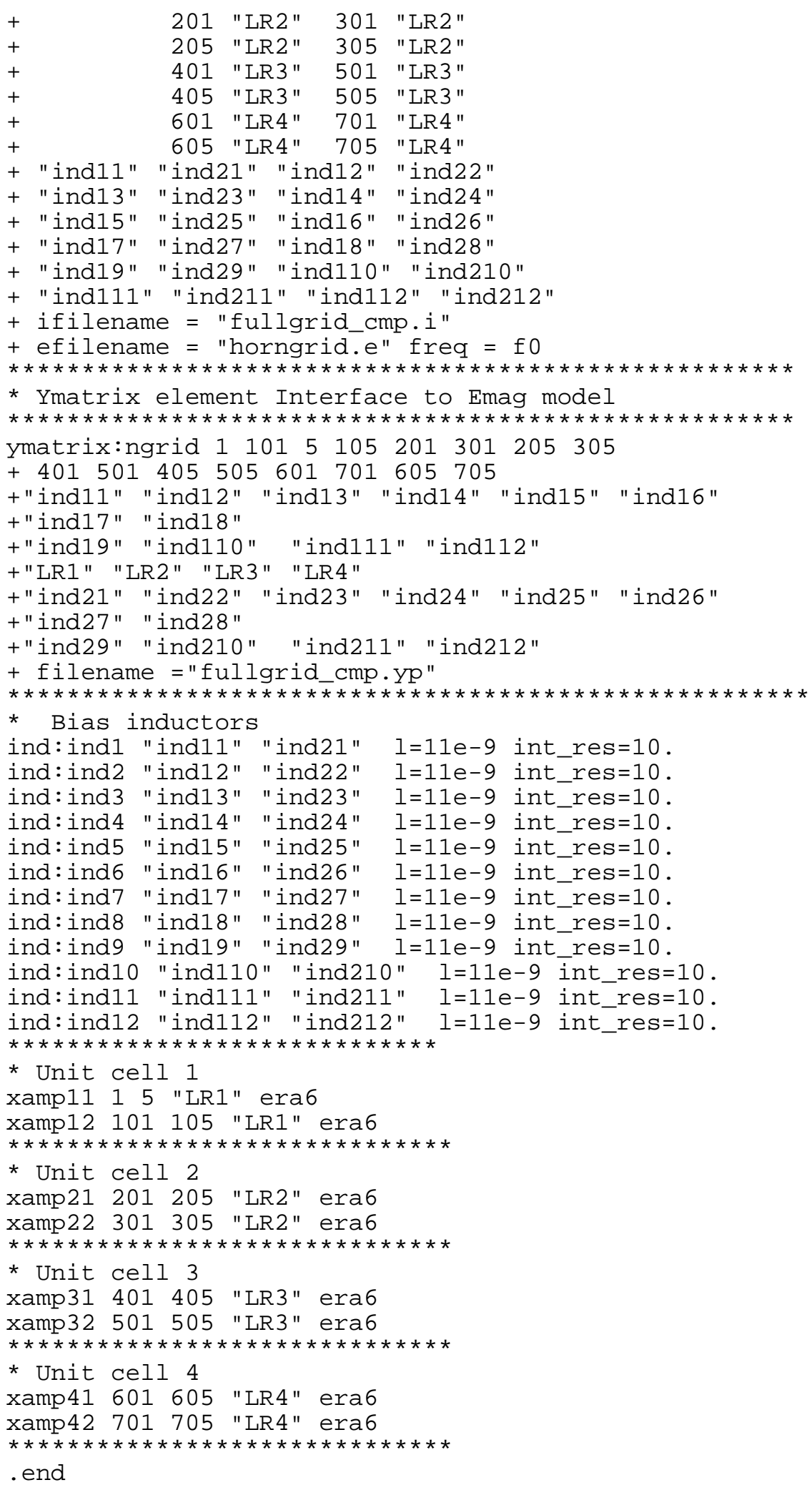

Figure 9. Transim netlist for the grid amplifier circuit of Figure 7 . 